\title{
The Research of Supplier Quality Based on the Supplier Relationship: Theoretical Definition and Construction of Evaluation Dimension
}

\author{
Changhong Tang ${ }^{1}$, Xiaorong $\mathrm{Li}^{2}$, Xiaohua $\mathrm{Lv}^{2}$ \\ ${ }^{1}$ Logistics School, Beijing Wuzi University, Beijing, China \\ ${ }^{2}$ Graduate Department, Beijing Wuzi University, Beijing, China
}

Email address:

tangchanghong@bwu.edu.cn (Changhong Tang), lixiaorong426@icloud.com (Xiaorong Li), xhalw2012@163.com (Xiaohua Lv)

\section{To cite this article:}

Changhong Tang, Xiaorong Li, Xiaohua Lv. The Research of Supplier Quality Based on the Supplier Relationship: Theoretical Definition and Construction of Evaluation Dimension. Science Journal of Business and Management. Vol. 4, No. 4, 2016, pp. 108-113.

doi: $10.11648 /$ j.sjbm.20160404.11

Received: June 1, 2016; Accepted: June 13, 2016; Published: June 30, 2016

\begin{abstract}
In recent years, enterprises are facing the competition among supply chains rather than enterprises. Quality is the foundation of enterprises. Suppliers play significant role in the supply chain and the quality of supplier relates to the stability of the supply chain. In this paper, the concept of the supplier quality is elaborated firstly on the basis of the related concepts. Then, considering the distinct extent relationship between the purchaser and supplier, supplier quality is divided into quality performance, quality ability and quality organization three dimensions. Besides, on the basis of the quality management and the evaluation of supplier, the concept of each dimension is elaborated. The construction of the evaluation dimensions is conducive to procurement the problem of excessive or insufficient evaluation. In addition, enriching the theory of the supplier quality of enterprises implementation.
\end{abstract}

Keywords: Supplier Relationship, Supplier Quality, Evaluation Dimension

\section{Introduction}

The famous master of quality management Joseph Juran once said, if the $20^{\text {th }}$ century is the century of efficiency, the $21^{\text {st }}$ century is the century of quality. [1] With the integrating development of global economic, enterprises facing the competition from many aspects today, the quality is the life of the enterprises. It is one of the core competitiveness of enterprises. The concept of quality usually refers to the inherent characteristics' extent of tangible or intangible products meeting the requirements. Tangible products usually including the hardware such as mechanical parts, mobile phone. As well as natural material such as oil, natural gas. With the deepening research, the applicability of the intangible products such as service, information is also given the concept of quality. In this paper, the concept and the evaluation content of the supplier quality are mainly discussed.

Supplier quality contains the quality of product, but it is not limited to this. With putting forward big quality and the higher attention of process, the research of supplier quality gradually involving the process of product, operating condition, service, organizational culture and so on. Especially since the Malcolm Baldrige National Quality Award has been widely spread, quality is replaced by excellent gradually. Now, companies not only need to hold its core competitive advantages, but also care for the suppliers. It is necessary for companies to find the proper suppliers effectively. Evaluating suppliers and ensuring supplier quality are the basic process of selecting suppliers. Prior to this, the analysis of the procurement of goods and supplier relationship analysis is also essential. In this paper, considering the different level of supplier relationships, the contents of supplier quality evaluation is divided into three dimensions, and the concept is briefly described. In this paper, the research enriching the related theory of supplier quality evaluation and provided an evaluating basic structure for companies. 


\section{The Related Concepts of Supplier Quality}

\subsection{Quality and Quality Management}

With the development of social productivity, quality management gradually becomes more and more important. According to the International Organizational for Standardization ISO9000: 2000, quality is defined as the totality of features and characteristics of a product or service that bear on its ability to satisfy given needs. [2] Quality features including physical and chemical properties of mechanical properties such as hardness, strength, time characteristics of durability, reliability, sensory characteristics of the visual and aesthetic value and so on. Understanding the quality should pay attention to its broad, relativity and timeliness. Generalized concept not only includes the quality of the tangible and intangible products, but also the process quality and system quality management. Relativity refers to distinct requirements will be putted forward by different people on the different point of views. Timeliness refers to the expectations of the demand requirements is not fixed for ever, the requirement of quality and the relevant work is also varies with time under the same related parties in different period.

Quality management is a series of related activities of planning, organizing, coordinating and controlling to achieve the quality goals of the organization. The quality management activities started in the $20^{\text {th }}$ century, according to the difference of the standing view and way, the solution of the quality problem was different. The modern quality management is divided into three periods, testing quality period, statistical quality period and total quality management period. Three phases aim to the product, process and management system respectively. From 30 in twentieth century, quality management concept was gradually emerging from the system perspective. The representative scholars are W. Edwards Deming, Joseph Juran, Kaoru Ishikawa and Crosby. Dr. A. V. Feigenbaum proposed total quality management (TQM) firstly in 1956. He advocated quality issue could not be confined to the quality of the finished product inspection and manufacturing process. Because the quality problem of manufacturing process in all accounts for only about $20 \%$ of the quality problems, $80 \%$ of the quality problem is not in the manufacturing process. The solution to the quality problem limited to the statistical methods is inadequate. It lays the foundation for the development of total quality management (TQM). In 1994 the international standard, TQM was defined as the follow. A long management way in an organization, the quality is took as the center with the foundation of the full participation, aiming at making customer satisfied and all members of the organization and social gain the benefits. Japanese quality management expert Kaoru Ishikawa epitomized quality management of all the company (referred to CWQC) summed up the characteristics of the whole company's upper to the staff quality management, from R\&D (Research and Development) to sale, personnel, financial and other issues, all of this participate in quality management. Quality management concepts and methods are not only limited to the production process, new product design, etc., but also use it to carry out business analysis, etc.

Practice of Chinese enterprises is summarized quality management as three full that are the whole process, all personal and comprehensive quality. The whole process means the process from the product's manufacturing, finally performance and the organization's management. The whole process is composed of multiple related links. Every link influences the final quality. Full Personal refers to all staff should participate in quality management, involving in the pursuit of excellence. Comprehensive means the enterprise should be seen as a whole system, departments are coordinating and achieving to the goal excellent.

Thus, quality management from the stage of back testing for product, after to the stage of beforehand prevent for statistical process quality control, finally to the stage of the whole system quality management. Now, quality management can be understood as the activities including plan, organize, coordination, control to pursue excellence, that's a new stage.

\subsection{Supplier Quality}

The current global economic is integrating and the social division of labor deepens gradually, companies increasingly focus on the development of the core competitiveness, and the non-core business is outsourced to others, the competition between enterprises has also risen to the competition between the supply chain, quality supervision has become one of the most important projects of enterprise. As the starting point or middlemen in the supply chain, supplier quality is a significant part in supply chain.

Supplier quality characteristics are restricted to tangible products in early description. Later, intangible products such as service, etc. are gradually involved in. Now, supplier quality involves tangible products, process, organization, activities, business's culture and so on. Accordingly, supplier quality has also including production quality, process quality, operating quality, culture quality etc. concept.

Above all, supplier quality can be summarized as the totality ability to satisfied or exceed the expectations and requirements putting forward by customer. [3] There are three aspects about understanding the definition. (1) On the basis of consistent, namely supplier ensure the product conforms to the customer's requirements firstly, that means the tangible products should meet the corresponding standards in the industry. Besides, the supplier should promise the intangible products such as corresponding service to remain consistent. (2) Customer's expectations now and future, that means the ability of supplier to improve the products or the service under the customers' requirement on the basis of consistent is necessary. Including the original design of products, to make processing and corresponding services. (3) The key performance area, supplier quality not only refers to the inherent quality characteristics, but also including suppliers in many key areas meeting the expectations and requirements of purchaser, including the supplier's financial operating conditions, the enterprise culture, etc. Supplier is considered 
as a quality management organization system. Totally, the aim of the supplier quality management is to send the right product to the right place with right quality, right quantity and right state in the right time. [4]

The research of supplier quality is mainly from supplier evaluation and quality evaluation. (1) Supplier evaluation. In the earliest research on the index system of supplier evaluation, the quality, delivery, past performance and other 23 criteria were put forward through the investigation and analysis of 170 purchasing managers and purchasing agents by GW Dickson. He divided the 23 criteria into four levels, which are extremely important, very important, generally important and a little important. Quality is considered as extremely important. Price, finance and technology are very important. The others are generally important and a little important. [5] Hathrall investigated a pharmaceutical industry, he found there are 8 indicators used for supplier evaluation generally, the order are as follows, quality, price, service, technical ability, financial capacity, credibility and communication, etc. [6] According to analysis the supplier relationship and supplier evaluation, Zhang Zhenzhu analysis the supplier evaluation mainly from five aspects, they are product competitive advantage, enterprise competitive advantage, enterprise external environment competition, enterprise compatibility and credibility of enterprises. [7]

Generally speaking, the supplier management index system mainly includes seven aspects. Respectively are quality, cost, delivery, service, technology, asset, people and process. In which the quality, price, delivery is applicable to all walks of life, reflecting the supplier's management performance directly. The rest of the indicators need to be analyzed specifically according to the industry. (2) Quality evaluation. Du Wenting aimed at auto parts suppliers, and the quality of auto parts suppliers was divided into three dimensions, which are production dimensions, raw materials to obtain dimensions and customer service satisfaction. [8] Alan proposed to analysis the supplier's process capability index to evaluate the supplier quality, and the bootstrap method is to be used to get its confidence interval. [9] Yang Jinlong summed up the product inspection pass rate, quality stability, fault rate, product capability, quality system, the level of detection and several aspects to evaluate the product quality. [10] Wu Wei evaluated the supplier quality from four aspects, respectively are product quality, service quality, process quality and management quality. He applied it to the supplier selection of electric tools. [11] Sun Peng divided the general parts supplier quality dimensions into quality ability dimensions, quality growth dimension, quality strategic dimensions and consistent quality dimensions. [12] Anjali Awasthi investigated that the poor quality product and the services leading to monetary loss and reduce the market reputation. He studied the quality from four perspectives. These are product quality, process quality, service quality and organizational quality. [13] Brian Fynes investigated the buyer-supplier relationship and quality management, and concluded that flexibility, dependability, and customer service need to consider from the manufacturing perspective if overall business performance is to improve. [14]

Quality management has experienced the process from local to whole. Testing and process control are aiming at products and process, that can be understood the local. The whole process, whole staff and the comprehensive quality management can be understood the whole. Supplier quality has also experienced the process from local to whole. Supplier has to satisfy the customer's recent requirement including products standard and the corresponding service at the beginning of the stage. After that, the purchaser will focus on the supplier's ability to satisfy the customer's expectation in the future. Finally, the key performance such as the operation and the finance will be focused on. That's the local to whole of the supplier quality.

\section{The Importance of Supplier Quality Evaluation}

The demand is cleared firstly by the purchaser when selecting and confirming supplier, and than according to the market supply analysis, the potential suppliers are confirmed. After that, through communicating to suppliers and sufficient information exchanging, possible suppliers are screened out. Then the final supplier is selected. Purchaser consumes part of resources from the preliminary selection supplier to the final procurement, once there are corresponding problems of the provided products, services, and commitment is not consistent, the loss will be caused to purchaser. Although the existing evaluation system of supplier is comprehensive, the research on the evaluation system of supplier relationship is less and inadequate.

Current researches of supplier evaluation mostly focus on quality, cost, delivery, etc. Actually, the difference value of the purchase product and the different development degree of the relationship between the supplier and the purchaser will affect the evaluation of the supplier. Such as the evaluation of the standardized conventional products and the key products is obviously distinct. The same evaluation system to the conventional goods is unnecessary, but to the key products is not sufficient. Although there is quality aspect of a purchaser assess suppliers, but in the quality evaluation process, the index usually stay in the current quality situation, common guidelines for the evaluation of product failure rate, return rate, and quality assurance system. Product's failure rate, return rate, quality assurance system, etc. are common evaluation criteria. [15] These indicators can reflect the quality of the product at the moment, but for those who want to cooperate with suppliers further, these indicators are not sufficient. Therefore, considering the relationship between purchaser and supplier, quality evaluation is divided into three dimensions in this paper from the perspective of quality, and the comprehensive quality management is the guiding ideology. Besides, the content in each dimension is elaborated. 


\section{Analysis of Supplier's Quality Dimension Under the Relationship Between the Supplier and Purchaser}

\subsection{The Development of Supplier Relationship}

Relationship can be defined as contact or cooperation. Relationship refers to the interaction between individuals, organizations, and groups inside and outside the organization. [16] In the recent market competition, the most ideal state is that both supply and demand are cooperative partners.

Management of supplier relationship began in the (1961) found of bullwhip effect phenomenon by Forrester When the demand fluctuating, [17] the respond of the supplier is not timely and that led to the supply and demand does not match. In the supply chain, relationships involve a wider network of relationships between the buyer, supplier, and competitive business. Supplier relationship management is a new theory based on the theory of supply chain management to deal with the relationship between customers and suppliers. Supplier relationship takes the guide of win-win, and the mutually beneficial strategic partnership is the highest goal. Supplier relationships develop from the traditional short-term trading to the long-term stability direction. The following table 1 shows the evolution of supplier relationship.

Table 1. Evolution of supplier relationship.

\begin{tabular}{|c|c|c|}
\hline & Traditional supplier relationship & Supplier relationship based on the supply chain \\
\hline $\begin{array}{l}\text { The subjects the purchaser } \\
\text { concerned about }\end{array}$ & $\begin{array}{l}\text { The price and quality of the products, } \\
\text { delivery and so on. }\end{array}$ & $\begin{array}{l}\text { The price, quality, delivery, the ability of the process, Reliability, operating } \\
\text { conditions, innovative design and other enterprise's overall operating conditions. }\end{array}$ \\
\hline Supplier's characteristics & $\begin{array}{l}\text { The quantity of supplier is much and } \\
\text { changeable }\end{array}$ & The quantity of supplier is less and stable. \\
\hline Contract nature & Short term trading & Long term cooperation agreement \\
\hline
\end{tabular}

\subsection{Classification of Purchasing Goods}

However, it is not necessary for all suppliers to develop into a long-term cooperative relationship with the purchaser, the value of purchasing goods and the risk of supply market is different. The quality level that purchaser pay attention to is also different. In this paper, according to the risk level of the supply market and the annual expenditure of two dimensions, the procurement of products are divided into four types, figure 1 shows the four types. [18]

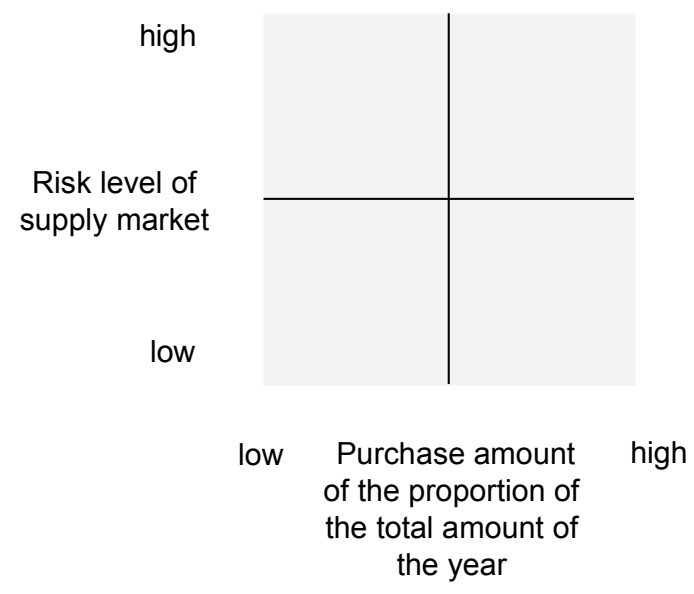

Figure 1. Classification of purchasing goods.

(1) Regular product's purchase amount and the supply market risk is low. This type of product is usually the standardized product. The requirement of the purchase to supplier's value is relatively low. So the purchase's attention to the trade is in general low, and the purchaser usually focus on the evaluation of the supplier's quality performance.

(2) Key product's purchase amount and the supply risk is high. The purchaser pay much attention to the product quality performance, as well as quality ability quality culture is all focused on. [19] Purchaser not only to ensure the product quality to meet the requirements of standard, but also to fully understand the supplier's internal process ability and operating condition, quality culture enterprise concept, so that the purchaser can guarantee the long-term cooperation with the supplier.

(3) Bottle neck product's purchase amount is low and the supply market risk is high. The leveraged product's purchaser amount is high and the supply market risk is low. Both of the two products are focused on the quality performance and the quality ability. Through understanding and coordinating with the supplier, the purchase and the supplier will decrease the purchasing cost.

\subsection{Evaluation Dimensions of Supplier Quality}

Supplier quality runs through the whole process of quality formation and implementation, which covers all aspects of the whole operation organization, the potential process capability and the quality of the results. According to these different types of products, the requirement to suppliers is also different. In this paper, the supplier quality evaluation content is divided into three dimensions, namely the quality performance dimensions, quality ability dimension and quality organization dimension, corresponding to the cooperative degree of purchaser and supplier, respectively the short-term, medium-term and long-term three stages.

(1) Quality performance

The supplier mainly concerned about the supplier's quality performance in the current period on short-term. Supplier's quality performance can be understood as excellent quality and excellent service. The products function should meet the 
requirement. Service including the prophase service and after sales service, the supplier's prophase service commitment, and the consistency of the service execution. Besides, price is the characteristic of economy in the concept of quality. The price of the product should in a proper range.

(2) Quality ability

In addition to the supplier's quality performance, the purchaser should pay close attention to the supplier's quality ability. Process determines product, correspondingly, the quality of the supplier's ability determines its quality performance. Therefore, the supplier's quality ability can be understood as the supplier's quality status in the process of providing qualified products and services. The quality capability is mainly aimed at the supplier's product forming process. The process is defined as a group of interrelated or interacting activities that can be converted to an output. Three scales can measure the quality of process: they are effectiveness, efficiency and adaptability. The effectiveness is the satisfied extent of product and service provided by the supplier. The design of supplier prophase process is the deciding factor. Efficiency refers to the relationship between the results of the process and the resources used. In the practice of quality management, there are many tools and methods of quality management, effective quality management tools and methods can make the process more efficient and less resource consumption. Adaptability refers to the degree of the effect and efficiency of the process in the face of various changes with the passage of time. In the course of the formation of products and services, there will be a variety of unexpected problems and failure; they are the hidden dangers of the quality. In the process of product design and maintenance, the reliability analysis method is usually used in failure mode and effect analysis (FMEA) and fault analysis tree.

(3) Quality organization

In addition to the current quality performance and quality capabilities, the purchaser need to pay attention to the quality of the supplier's organization if the purchaser and suppliers want to establish a long-term strategic partnership. Deming pointed in the System Drives Behavior, when there is a problem with the product or service, more than $85 \%$ of the problems can be attributed to the management system itself, and only $15 \%$ can be attributed to the products and services provided directly or execution. All the human, material and financial resources of the supplier are in the same organization system. In this system, there are human resources management system, production management system, financial management system and so on. A powerful and efficient organization to make full use of these resources with the greatest effect. The supplier's quality organization determines the supplier's quality ability; the supplier's quality ability decides the supplier's quality performance.

Through the elaborate of the supplier quality, the evaluation of supplier quality is shown in figure 2. There are three dimensions of the evaluation, and the considered factors are as follows. Under the quality performance dimension, the technical is the measurement of the product function. The service guarantee is the satisfaction degree of the related customer. Price stabilization is the factor considered from the expense. In quality ability dimension, the conformance is the measurement of the effectiveness above. And the process factor is the measurement of the efficiency and adaptability. The production factor points to the total produce guarantee ability, including safety production, field management and so on. In the quality organization dimension, the operation capital, management team and the strategic planning is the considering factors.

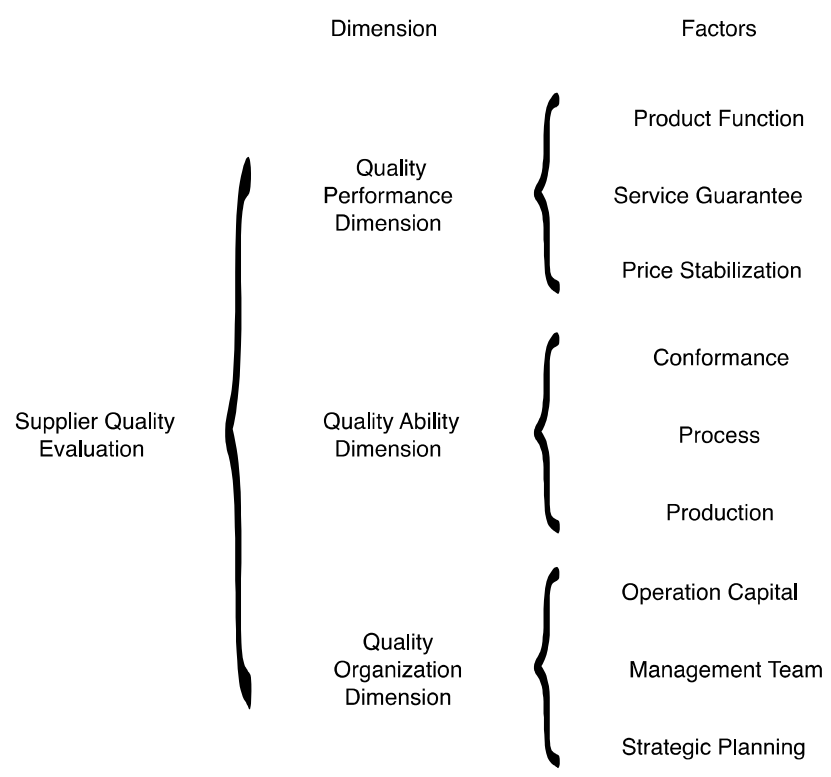

Figure 2. The supplier quality evaluation dimension and the content.

Purchasers and suppliers focus on the quality performance dimensions, mainly from the product function, service guarantee and price stabilization. The corresponding evaluation indexes are qualified products proportion, delivery commitment, after-sale protection and so on. When they cooperate in the medium term, the purchaser pays much attention to the supplier's manufacturing process ability in order to guarantee whether there is the potential for long-term development, that is the quality capability dimension. The corresponding evaluation indexes include the production capacity, process design, process inspection, failure control and so on. When the purchaser decided to cooperate with the supplier in a long term, the purchaser will pay attention to the quality organization dimension besides the two above dimension. The purchaser will pay attention to the index such as the operational status, quality concept, and development potential etc. In the different stage of cooperation, the purchaser will pay attention to the distinct quality content.

\section{Concluding Remarks}

Quality is the life and the core competitiveness of enterprise; it related to the development and survival of enterprises. This paper first describes the concept of supplier quality, than starts from the supplier relationship and according to the purchaser products and the classification, the quality content is divided into three dimensions. The content of each dimension is also 
explained. This paper enriches the relevant theory of supplier quality evaluation under different conditions. In addition, the research is mainly on the theory, and the practice in the enterprise will be the further research content.

\section{References}

[1] Chen yuntao. Quality Management [M]. Beijing: Tsinghua University press, Beijing Jiaotong University press, 2008, 1, 21 .

[2] Song yuqing. Logistics Enterprises to Implement the 2000 Edition of the ISO 9001 Standard Guide. Beijing: China Standard press, 2005, 6-7.

[3] Robert B. Handfield, Robert M. Monczka. Sourcing and Supply Chain Management, Fifth Edition [M]. Beijing: Electronic Industry Publishing House. 2014, 214.

[4] Wu Zhiyin, Research on supplier selection evaluation of auto parts based on product quality, [D], Hunan University, 2012.

[5] Dickson GW. An analysis of vendor selection systems and decisions, [J], Journal of Purchasing, 1966, 2: 5-17.

[6] Hatherall D. A. Purchasing in the pharmaceutical industrial, [D]. Unpublished M. Phil. Thesis, Department of management Science, Lancaster University, U.K. 1988.

[7] Zhang Zhenzhu, Analysis of supplier relationship management based on Supply Chain Environment, [D], Tianjin University, 2005.

[8] Du Wenting, Research on the quality evaluation system of Shanghai Volkswagen Parts Supplier, [D]. Shanghai Jiao Tong University, 2009.

[9] Lv Jie, Research on supplier quality supply capability under the condition of mixed quality characteristics, [D], Shandong University, 2007.

[10] Yang Jinlong, Research and application of the existing supplier quality evaluation system of Shanghai General Motors, [D]. Shanghai Jiaotong University, 2009.

[11] $\mathrm{Wu}$ Wei, Research on the evaluation of the supplier quality management capability of the electric tools [D]. Nanjing University of Aeronautics \& Astronautics. 2013.

[12] Sun Peng, The construction of supplier quality evaluation dimension structure under the environment of supply chain management, [D], Harbin Engineering University, 2014.

[13] Anjali Awasthi, Supplier Quality Evaluation Using a Fuzzy Multi Criteria Decision Making Approach, [J]. Complex System Modeling and Control Through Intelligent Soft Computations. 2014 (30): 195-219.

[14] Brian Fynes, The moderating effect of buyer-supplier relationships on quality practices and performance, [J]. International Journal of Operations \& Production Management. 2002, (6): 589-613.

[15] Yu Kunpeng. Research on some problems of production supply chain vulnerability. [D]. Chongqing University, 2013.

[16] Kenneth Lysons, Brian Farrington. Purchasing and Supply Chain Management, 7 e. Beijing: Electronic Industry Publishing House, 2007, 176-177.

[17] Luo Shoujian. Supplier Relationship [M]. Shanghai: Shanghai University of Finance and Economics press, 2009, 11-12.

[18] Tang Changhong. Agile: the 10 role of Supply Chain Director [M]. Beijing: China Development press, 2010, 67.

[19] Sun Peng. Research on Quality Evaluation of Product General Parts Supplier Under SCM Environment. [D]. Harbin Engineering University, 2014. 